\title{
Research on Oversight Mechanisms of Online Car-Hailing Platforms Based on Three-Party Game
}

\author{
Weiye Luo \\ School of Management, Jinan University, Guangzhou, China \\ Email: luoweiye0760@163.com
}

How to cite this paper: Luo, W.Y. (2019) Research on Oversight Mechanisms of Online Car-Hailing Platforms Based on Three-Party Game. Journal of Human Resource and Sustainability Studies, 7, 24-36.

https://doi.org/10.4236/jhrss.2019.71003

Received: February 19, 2019

Accepted: March 5, 2019

Published: March 8, 2019

Copyright $\odot 2019$ by author(s) and Scientific Research Publishing Inc. This work is licensed under the Creative Commons Attribution International License (CC BY 4.0).

http://creativecommons.org/licenses/by/4.0/

\begin{abstract}
Online car-hailing has become a very popular travel mode in China in recent years. In order to provide decision support for government so that the car-hailing industry can develop better, the present paper built a mathematical model among the government, online car-hailing platforms and consumers based on game theory. After analyzing the eight strategies of Nash equilibrium and mixed strategy Nash equilibrium, it has been found that the government is supposed to make safety codes, conduct rewards and punishment system, define responsibility for both platforms and consumers clearly and enhance publicity to keep the industry developing steadily.
\end{abstract}

\section{Keywords}

Online Car-Hailing, Three-Party Game, Nash Equilibrium, Oversight Mechanism

\section{Introduction}

Since the Internet rental service was launched in China in 2012, it has been developing at an unprecedented speed [1]. According to a survey done by iiMedia Research [2], the number of users of online car-hailing in China reached 362 million in 2016. Even under the influence of policies and other macro-environment, it is estimated that 308 million users will participate in the use of online car-hailing by 2018. It can be seen that online car hailing has become one of the important ways for people to make a short-distance travel or commute.

However, with the rise of online car-hailing, many problems have followed, among which the most prominent one is the safety problem. Due to insufficient supervision and lack of legal standards, crime events caused by online car-hailing such 
as fighting, robbery and rape, often occur. In January and April, 2016, Shenzhen security department has received 439 alerts related to online car-hailing. It can be seen that the problem of online car-hailing supervision cannot be ignored [3].

On July 28, 2016, the ministry of transport and other departments jointly issued the "guidance opinions on deepening reform and promoting the healthy development of taxi industry" and "interim measures on the management of online booking taxi business services", which clarifies the legal status of online car-hailing, and China has become the first country in the world to legalize online car-hailing. However, should we strictly control online car-hailing? These questions are worth exploring.

\section{Literature Review}

According to "report on Chinese language and living conditions (2016)" [4], taxis are divided into cruise taxi and online taxi booking. Among them, online taxi booking, also known as online taxi booking, refers to a vehicle that can be booked through the mobile Internet and charged by mileage.

Since the birth of car-hailing, scholars have been discussing its influence and regulation. Mao S.L. analyzed government regulation and social impact from the perspective of legalization of online car-hailing, and believed that the recognition of legal status of online car-hailing could effectively improve the chaos of "black car", but this increased the difficulty of government management [5]. Gao Y. discussed the influence of online car-hailing on traffic operation. Their study took Beijing city's online car-hailing users as samples and studied the change of their travel mode choice quantitatively [6]. It is found that online car booking is an important cause of urban traffic congestion.

On the other hand, many literatures focus on the supervision of online car-hailing. Li analyzed the safety risks of online car-hailing and proposed corresponding countermeasures for the safety problems of online car-hailing passengers [7]. He pointed out that there are important operational loopholes in car-hailing, including low threshold for drivers, loopholes in vehicle examination and low safety awareness of passengers. The paper also argues that the government should help online car-hailing platforms improve the entry threshold for drivers and vehicles, and strengthen the safety awareness of consumers. Wang J. studied the development process of online car-hailing, made an in-depth discussion on its regulatory difficulties, and proposed solutions to administrative supervision [8]. She believes that quantity control, price control and improvement of vehicle and driver standards will be conducive to social security and stability. Xue Z.Y. gave useful suggestions on the quantity control of online car-hailing by combining the central and regional sample studies. This paper holds that the government should gradually deregulate online car-hailing, and at the same time, the government should give full play to the market power, strengthen the management of regulatory bodies and prevent their abuse of power [9]. Mei J. divided online car-hailing into full-time online car-hailing and 
part-time online car-hailing, and believed that different types of online car-hailing should develop different regulatory measures [10]. Zhang Y.N. discussed the lack of safety supervision of drivers and the fake use of information in connection with the existing two major regulatory problems of online car-hailing, and put forward regulatory Suggestions [11].

To sum up, previous literatures have conducted a wide range of discussions on online car-hailing, mainly from a qualitative perspective to analyze the impact of online car-hailing on society and propose solutions to existing problems. However, there are few articles on the analysis of the regulation of car-hailing by game theory. Based on this, this paper uses game theory to analyze the game among the government, online car-hailing platform and consumers, so as to design an effective regulatory mechanism for the government and provide a new perspective for the analysis of problems related to online car-hailing.

\section{Building a Tripartite Game Model}

\subsection{Description and Hypothesis}

In this paper, a game model is established for the three parties: the government, online car-hailing platform and consumers. Among them, government mainly refers to local governments and institutions or organizations that can formulate policies related to online car-hailing. Online car-hailing platform refers to the online and offline travel platform that can integrate resources between online car-hailing and consumers' travel demands. Consumers refer to people who want to meet their travel needs without using their own means of transport.

In order to facilitate analysis, the government adopts the strategies of "regulate" and "not regulate" in this model. Among them, the "regulate" strategy refers to that the government invests certain financial, human and material resources to supervise the safety and reliability of online car-hailing. The "not regulate" strategy means that the government does not impose mandatory requirements on the safety of online car-hailing, and specific access rules are formulated by each online car-hailing platform according to market.

The strategies adopted by car-hailing platforms are "strict regulatory measures" and "loose regulatory measures". Among them, "strict regulatory measures" are aimed at providing consumers with a high safety driving experience. In this way, the car-hailing platform will strictly check the drivers and cars and continuously track and supervise them in the subsequent operation, which will increase their operating costs. However, "loosely regulatory measures" refer to the fact that car-hailing platforms do not specify strict regulations or, although strict regulations have been formulated, do not strictly follow the established rules. Although the operating cost of the online car-hailing platform is reduced, it cannot provide consumers with high-security ride services.

Consumers' strategies are "use" and "refuse". "Use" strategy refers to the use of the mobile Internet to make car reservations to meet their travel needs. The "refuse" strategy refers to that consumers refuse to use the Internet car booking 
service and meet their travel needs through other means of travel.

This model has the following assumptions:

- Rational man hypothesis: it is assumed that all three parties of the model are rational men, and their goals are to maximize their own profit, and take this as the action criterion. For the government, the purpose is to pay the least cost to minimize the negative impact on society. For car-hailing platforms, the goal is to maximize their profits. Consumers maximize utility.

- The model assumes that the tripartite game is a static game with complete information, that is, each participant has accurate information about the characteristics, strategies and payment functions of other participants, and that Nash equilibrium solution can be obtained at last.

- The problem that the government is most concerned about is the negative impact of society, and the regulation of online car-hailing can solve the problem that the government is concerned about.

\subsection{Model Parameters}

\subsubsection{Parameters Related to the Government}

The government's strategy is to regulate and not regulate, and for the situation of regulation, the government will pay regulatory cost $C$. If the car-hailing platforms can implement strict regulatory measures, the government will reward $R$. On the contrary, if the car-hailing platforms do not implement strict regulatory measures, the government will impose penalties $F$ on them. When car-hailing platforms fail to implement strict regulatory measures, the government will suffer costs brought by negative social influences $N$. For the government to adopt a non-regulatory strategy, the government does not need to pay regulatory costs, nor does it reward or punish the car-hailing platform, but it may still be negatively affected by the society.

\subsubsection{Parameters Related to the Car-Hailing Platform}

The alternative strategies for car-hailing platforms are: "strict regulatory measures" and "loose regulatory measures". When strict supervision measures are taken, the revenue of the car-hailing platform is $I_{h}$, so the cost is $C_{h}$. In the case of loose supervision, the profit is $I_{l}$ and the cost is $C_{l}$.

\subsubsection{Parameters Related to the Consumers}

Consumers can choose to use or refuse to use the online car booking service. When the car-hailing platform takes strict supervision measures and consumers use the car-hailing platform, the utility of consumers is $U_{h}$. When the online car-hailing platform does not take strict supervision measures and consumers use online car-hailing, the utility of consumers is $U_{l}$. When consumers do not use the online car booking service, the utility of consumers is 0 .

\subsection{Revenue Matrix}

Based on the above assumptions and parameters, the payment matrix based on 
the tripartite game among the government, online car-hailing platform and consumers can be obtained. Figure 1 and Figure 2 show the payment matrix of online car-hailing platform and consumers under different strategies selected by the government respectively. Among them, the earnings of all parties are separated by commas, which represent the government, online car-hailing platform and consumers respectively.

\section{Analysis of the Tripartite Game Model}

\subsection{Analysis of Pure Strategy Nash Equilibrium}

According to the payment matrix in Figure 1 and Figure 2, the Nash equilibrium solution method of the three-party game is used for analysis [12], as shown in the following Table 1.

Firstly, the combination of strategy 1 (Regulate, Strict, Use) is irrational, which conditions are $\left(I_{h}-I_{l}\right)+R+F>C_{h}-C_{l}, U_{h}>0, F>C$. It shows that it is profitable for enterprises to implement strict supervision measures than not to implement them. Therefore, enterprises will choose the strategy of "implementing strict supervision". For consumers, they will also choose to use the online car-hailing service because it will have a positive effect. However, for the government, $F>C$ indicates that the penalty income of the government is greater than the regulatory cost it pays. Here, enterprises will choose a strict regulatory

Consumers

\begin{tabular}{l|c|c|} 
& Use & Refuse \\
\cline { 2 - 3 } Strict Regulatory Measures & $-C-R, I_{h}-C_{h}+R, U_{h}$ & $-C-R,-C_{h}+R, 0$ \\
\cline { 2 - 3 } Loose Regulatory Measures & $-C+F-N, I_{l}-C_{l}-F, U_{l}$ & $-C+F-N,-C_{l}-F, 0$ \\
\cline { 2 - 3 } & &
\end{tabular}

Figure 1. Payment matrix when the government chooses "regulate".

\begin{tabular}{l|c|c}
\multicolumn{2}{c}{ Consumers } \\
\cline { 2 - 3 } Strict Regulatory Measures & Use & $0,-C_{h}, 0$ \\
\cline { 2 - 3 } Loose Regulatory Measures & $-N,-C_{l}, 0$ \\
\cline { 2 - 3 } & $-N, I_{l}-C_{h}, U_{h}, U_{l}$ & $-N$
\end{tabular}

Figure 2. Payment matrix when the government chooses "not regulate".

Table 1. Nash equilibrium results of pure strategy.

\begin{tabular}{ccc}
\hline No. & Conditions & Pure Strategy \\
\hline 1 & $\left(I_{h}-I_{l}\right)+R+F>C_{h}-C_{l}, U_{h}>0, F>C$ & Regulate, Strict, Use \\
2 & $\left(I_{h}-I_{l}\right)+R+F<C_{h}-C_{l}, U_{l}>0, F>C$ & Regulate, Not Strict, Use \\
3 & $R+F>C_{h}-C_{l}, U_{h}<0, F>C$ & Regulate, Strict, refuse \\
4 & $R+F<C_{h}-C_{l}, U_{l}<0, F>C$ & Regulate, Not Strict, refuse \\
5 & $C_{h}-C_{l}<I_{h}-I_{l}, U_{h}>0$ & Not Regulate, Strict, Use \\
6 & $I_{h}-I_{l}<C_{h}-C_{l}, U_{l}>0, F<C$ & Not Regulate, Not Strict, Use \\
7 & $C_{h}<C_{l}, U_{h}<0$ & Not Regulate, Strict, refuse \\
8 & $C_{l}<C_{h}, U_{l}<0, F<C$ & Not Regulate, Not Strict, refuse \\
\hline
\end{tabular}


strategy, so the penalty income should be less than the government cost $C$, and the combination of this strategy is unreasonable due to the contradiction of conditions.

Secondly, the combination of strategy 3 (Regulate, Strict, refuse) is also irrational, which conditions are $R+F>C_{h}-C_{l}, U_{h}<0, F>C$. It means that enterprises will choose strict regulatory measures in consideration of the profitability of implementing strict regulatory measures. However, consumers will not choose to use online car-hailing because the utility obtained by choosing online car-hailing is less than 0 . Similar to strategy $1, F>C$ is only established when many car-hailing platforms do not carry out strict supervision, but in this situation, considering the cost, all car-hailing platforms will carry out strict supervision, which makes it unreasonable.

Then, for strategy combination 5 (Not Regulate, Strict, Use), although it is the ideal result, it is not reasonable, which condition are $C_{h}-C_{l}<I_{h}-I_{l}, U_{h}>0$. It shows that due to the cost, car-hailing platforms will voluntarily choose to implement strict supervision measures, because they can obtain more benefits by doing so. In this case, consumers are also willing to choose this mode of travel, because its utility is positive; Due to cost considerations and knowing that car-hailing platforms will voluntarily carry out strict supervision, the government will not impose additional and redundant supervision on such platforms. However, this is not the case in reality. When the car-hailing platform chooses to conduct strict supervision, it needs to pay more costs. Therefore, in the current situation that the online car-hailing industry is not very mature, strict supervision means more resources are needed for the online car-hailing platforms.

Then, policy combination 7 (Not Regulate, Strict, refuse) is obviously unrealistic, and its conditions are: $C_{h}<C_{l}, U_{h}<0$. That is to say, the government does not regulate, and consumers, realizing that the use of online car-hailing platforms will not bring positive effects, will not use them. However, online car-hailing platforms will still choose to establish more costly strict supervision measures.

Finally, for the strategy combination 2, 4, 6 and 8, since the government conforms to the hypothesis of rational man, it will pursue the maximization of its own interests, that is, the government will pursue the lowest negative impact on society and the lowest cost, so the government will tend to choose the "non-regulatory strategy". Similarly, for car-hailing platforms, if they choose to implement strict regulatory measures, the government will choose the "non-regulatory" strategy to save its own costs. And when enterprises find that the cost of implementing strict measures is higher than that of not implementing strict measures, in order to save costs, enterprises will not carry out strict supervision. Finally, consumers will make strategic choices based on their own utility value. When the utility of the car-hailing platform is less than 0 , consumers are more inclined to not use it and choose other ways to meet their travel needs. According to the above discussion, strategy combinations 2, 4, 6 and 8 will not 
naturally become pure strategy Nash equilibrium.

To sum up, there is no pure strategy Nash equilibrium in this model. Therefore, in order to reach a stable state, the model will choose mixed strategy to reach an equilibrium state.

\subsection{Analysis of Mixed Strategy Nash Equilibrium}

It can be known from the above analysis that there is no pure strategy Nash equilibrium in this model, so mixed strategy Nash equilibrium analysis should be carried out in this model to obtain equilibrium results.

$\alpha, \beta, \gamma$ respectively represent the probability that the government takes supervision measures, the probability that car-hailing platforms take strict supervision, and the probability that consumers use car-hailing. Thus, the mixed strategies of the three parties can be obtained as follows:

For the government:

$$
\sigma_{G}=(\text { regulate }, \text { not regulate })=(\alpha, 1-\alpha), \alpha \in(0,1)
$$

For online car-hailing platform:

$$
\begin{aligned}
\sigma_{B} & =(\text { strict regulatory measures, loose regulatory measures }) \\
& =(\beta, 1-\beta), \beta \in(0,1)
\end{aligned}
$$

For consumers:

$$
\sigma_{C}=(\text { use, refuse })=(\gamma, 1-\gamma), \gamma \in(0,1)
$$

The following is to use the payment equivalence method [13] to solve the Nash equilibrium of hybrid strategy:

\subsubsection{Analysis of the Government}

Where $\alpha=1$ represents the effectiveness of the government under regulatory measures:

$$
\begin{aligned}
V_{G}(1, \beta, \gamma)= & \beta[\gamma(-C-R)+(1-\gamma)(-C-R)] \\
& +(1-\beta)[\gamma(-C+F-N)+(1-\gamma)(-C+F+N)] \\
= & \beta(N-F-R)+F-C-N
\end{aligned}
$$

Set $\alpha=0$, indicating the effectiveness of the government under non-regulatory measures:

$$
\begin{aligned}
& V_{G}(0, \beta, \gamma)=\beta[\gamma(0)+(1-\gamma)(0)]+(1-\beta)[\gamma(-N)+(1-\gamma)(-N)]=N(\beta-1) \\
& \text { If } V_{G}(1, \beta, \gamma)=V_{G}(0, \beta, \gamma) \text {, then: } \\
& \qquad \beta(N-F-R)+F-C-N=N(\beta-1)
\end{aligned}
$$

obtained:

$$
\beta=\frac{F-C}{F+R}
$$

\subsubsection{Analysis of Online Car-Hailing Platform}

Let $\beta=1$, indicating the effectiveness of the car-hailing platform under strict 
regulatory measures:

$$
\begin{aligned}
V_{B}(\alpha, 1, \gamma)= & \alpha\left[\gamma\left(I_{h}-C_{h}+R\right)+(1-\gamma)\left(-C_{h}+R\right)\right] \\
& +(1-\alpha)\left[\gamma\left(I_{h}-C_{h}\right)+(1-\gamma)\left(-C_{h}\right)\right] \\
= & \alpha R+\gamma I_{h}-C_{h}
\end{aligned}
$$

Let $\beta=0$, indicating the effectiveness of the car-hailing platform under the non-strict supervision measures:

$$
\begin{aligned}
V_{B}(\alpha, 0, \gamma)= & \alpha\left[\gamma\left(I_{l}-C_{l}-F\right)+(1-\gamma)\left(-C_{l}-F\right)\right] \\
& +(1-\alpha)\left[\gamma\left(I_{l}-C_{l}\right)+(1-\gamma)\left(-C_{l}\right)\right] \\
= & \gamma I_{l}-\alpha F-C_{l}
\end{aligned}
$$

If $V_{B}(\alpha, 1, \gamma)=V_{B}(\alpha, 0, \gamma)$ then:

$$
\alpha R+\gamma I_{h}-C_{h}=\gamma I_{l}-\alpha F-C_{l}
$$

obtain:

$$
\begin{array}{r}
\gamma=\frac{\alpha(R+F)+C_{l}-C_{h}}{I_{l}-I_{h}} \\
\alpha=\frac{C_{h}-C_{l}-\gamma\left(I_{h}-I_{l}\right)}{R+F}
\end{array}
$$

\subsubsection{Analysis of Consumers}

Let $\gamma=1$, indicating the utility of consumers using the car-hailing platform:

$$
\begin{aligned}
V_{C}(\alpha, \beta, 1) & =\alpha\left[\beta\left(U_{h}\right)+(1-\beta)\left(U_{l}\right)\right]+(1-\alpha)\left[\beta\left(U_{h}\right)+(1-\beta)\left(U_{l}\right)\right] \\
& =\beta\left(U_{h}-U_{l}\right)+U_{l}
\end{aligned}
$$

$\gamma=0$, indicating the utility of consumers who do not use the car-hailing platform:

$$
V_{C}(\alpha, \beta, 0)=\alpha[\beta(0)+(1-\beta)(0)]+(1-\alpha)[\beta(0)+(1-\beta)(0)]=0
$$

If $V_{C}(\alpha, \beta, 1)=V_{C}(\alpha, \beta, 0)$ then:

$$
\beta\left(U_{h}-U_{l}\right)+U_{l}=0
$$

obtain:

$$
\beta=\frac{U_{l}}{U_{l}-U_{h}}
$$

Based on the Nash equilibrium results of the above mixed strategy, the following is a detailed analysis of the government, online car-hailing platforms and consumers.

Firstly, the Nash equilibrium of the government's mixed strategy is analyzed.

From Equation (3) $\alpha=\frac{C_{h}-C_{l}-\gamma\left(I_{h}-I_{l}\right)}{R+F}$ we can know that $\left(C_{h}-C_{l}\right)$ is positively correlated with the probability of the government implementing the surveillance strategy, which also means that compared with not implementing strict supervision, the higher the cost of strict supervision of online car-hailing 
platforms, the more likely the government will choose to supervise them. Because the cost of strict supervision is too high for online car-hailing platforms, they tend not to implement supervision. For the government, the lack of strict supervision of online car-hailing platforms will cause the government to bear the negative social impact, so the government will choose to supervise online car-hailing platforms.

On the other hand, probability $\alpha$ of the government's supervision strategy is negatively correlated with $\left(I_{h}-I_{l}\right), R, F$. That is to say, the higher the revenue of car-hailing platforms under strict supervision, but the lower the revenue under non-implementation, the more likely the government is not to supervise; The higher the reward or penalty of government supervision for online car-hailing platforms, the less inclined they are to choose supervision. Because, when $\left(I_{h}-I_{l}\right)$ is larger, it indicates that the car-hailing platform is more motivated to implement strict supervision, because it can obtain more benefits. At this time, the government does not need to implement additional supervision on the car-hailing platform. However, the larger $R$ is, the higher the cost of government supervision will be. Therefore, in this case, the government is not inclined to supervise. Finally, the government will impose huge fines on online car-hailing platforms that do not carry out strict supervision, and any rational online car-hailing platform will choose to take strict supervision measures, so the probability of the government choosing to supervise will be reduced. It is worth mentioning that when consumers are more willing to use online car-hailing platforms, according to the equilibrium result, the government will reduce the probability of using supervision. But in real life, if more consumers choose to use it, the government will be more inclined to supervise the industry in order to reduce negative social impact and ensure social stability.

Secondly, the mixed Nash equilibrium results of car-hailing platforms are analyzed. From Equation (1) $\beta=\frac{F-C}{F+R}$ we can know that The probability of strict regulatory measures implemented by car-hailing platforms is positively correlated with government fine $F$. That is to say, if the government supervises online car-hailing platforms and imposes a larger fine on those platforms that do not carry out strict supervision, in consideration of the cost of violation, then online car-hailing platforms are more inclined to implement strict supervision. But when car-hailing platforms know the cost of government oversight, the picture is different. In order to supervise the industry, the higher the cost the government has to pay, the more likely the car-hailing platforms will not adopt strict supervision. This is because car-hailing platforms know that high costs will make the government give up supervision, so they do not need to adopt more costly strict supervision. Finally, if government incentives increase, car-hailing platforms are less likely to implement strict regulation. In this regard, it can be understood that the strict supervision of online car-hailing platforms is only for the purpose of obtaining high government rewards. Once the platforms are re- 
warded by the government, they will not continue to operate or implement strict supervision.

Combining Equation (4) $\beta=\frac{U_{l}}{U_{l}-U_{h}}$, it is easy to see that $\beta$ is positively correlated with $U_{h}$ and negatively correlated with $U_{l}$. In practice, it means that online car-hailing platforms tend to meet customer needs. When consumers get higher utility in a high-security environment, in order to attract consumers, online car-hailing platforms will choose strict supervision to meet customer needs, and vice versa.

Finally, the mixed strategy Nash equilibrium of consumers is analyzed. From Equation (2) $\gamma=\frac{\alpha(R+F)+C_{l}-C_{h}}{I_{l}-I_{h}}$ we can know that $\gamma$ is proportional to $\alpha, R, F,\left(I_{h}-I_{l}\right),\left(C_{l}-C_{h}\right)$. This means that, for the government, the more it regulates the industry, and consumers are more willing to use online car-hailing services when the benefits and costs of online car-hailing platforms are higher and lower under strict supervision. Because the government has supervision, and the platform can make profits from the operation, so that it can spontaneously improve the operation and improve the safety, both of which can improve the overall efficiency of the entire online car-hailing industry. In this case, a rational consumer is certainly willing to use the online car-hailing service. This is also the ideal situation in reality, which is beneficial to all three parties.

\section{Supervision Mechanism for Online Car-Hailing Platforms}

\subsection{Necessity of Supervision over Online Car-Hailing Platforms}

According to the Nash equilibrium analysis above, it can be known that a high security service environment of online car-hailing platform cannot be spontaneously formed. Because online car-hailing platforms are always profit-driven, once the government does not take regulatory measures, platforms tend to choose low and strict regulation that can reduce operating costs or even do not supervise the safety of online car-hailing. And because consumers choose according to their own utility, in reality, platforms often attract consumers to take online car reservation with hidden safety risks through price subsidies, so that social problems will continue to arise. Therefore, to maintain social stability, the government needs to effectively supervise the online car-hailing industry. Specifically, the government can construct the supervision mechanism at the two levels of online car-hailing platform and consumers.

\subsection{Positive Incentive Mechanism}

As for online car-hailing platforms, according to the Nash equilibrium of the above mixed strategy, as long as their benefits are increased and their costs are reduced, it can promote the platform to choose strict supervision of online car-hailing platforms, so as to create a good ride safety. Therefore, the government should formulate codes of practice for the online car-hailing industry to 
provide basis and convenience for future regulation. Secondly, check the online car-hailing platform according to the safety rules. If the enterprises pass the inspection, they will be rewarded. It is worth noting that the reward subsidy to the platform should not be too high, or some platforms may be operated for the purpose of swindling the reward. On the other hand, innovation in operation supervision of online car-hailing platforms is encouraged to reduce the supervision cost of the platforms and improve the supervision efficiency. The government can set up technological innovation awards for online car-hailing platforms and give certain awards to platforms that have successfully developed effective regulatory measures. The above two aspects are measures to reduce the supervision cost of online car-hailing platforms.

For consumers, it can increase the publicity of car safety. Inform consumers how to protect their life and property safety when using online car-hailing services. More importantly, let them know how to choose different online car-hailing services. Secondly, for online car-hailing platforms that have passed the government's safety inspection, the government can issue qualification certification to encourage consumers to choose online car-hailing platforms with safety qualification certification. The measures, aimed at consumers, are intended to boost revenues for platforms that adopt strict regulations.

Therefore, the core of building a positive incentive mechanism lies in that the platform is willing to implement strict supervision over online car-hailing by directly controlling the platform end and reducing its supervision cost. On the other hand, through the promotion of consumers, the revenue of those platforms that implement strict supervision can be improved, so as to improve the industry level.

\subsection{Negative Incentive Mechanism}

For online car-hailing platforms, first of all, those who fail to operate in accordance with the safety rules stipulated by the government will be fined heavily. The goal is to raise the cost of breaking the law on the platform. On the other hand, for consumers who have suffered losses due to the use of the platform's online car-hailing service, the government should clearly stipulate that the platform shall bear certain responsibilities. In this way, the responsibility of online car-hailing is bound up with that of the platform. Once online car-hailing causes losses to passengers, the platform is also liable for inadequate supervision.

For consumers, be clear about the dangers of using non-standard platforms. And let the consumers know that they choose the online car-hailing platform without safety qualification certification under the circumstance of knowing. In case of an accident, consumers also need to bear part of the responsibility.

Therefore, improving the illegal cost of the platform is the key to build a negative incentive mechanism. By increasing fines and clarifying responsibilities, the platform will implement strict supervision to enable consumers to choose plat- 
forms with high safety.

\section{Conclusions}

In this paper, a tripartite game model is constructed, solved and analyzed by applying the method of game theory. It is found that the model does not spontaneously form pure strategy Nash equilibrium. Therefore, each participant will select the pure strategy with a certain probability, that is, the mixed strategy. Through the analysis of Nash equilibrium of hybrid strategy, it is found that the government should guide or help the online car-hailing platform to reduce the regulatory cost, so as to help establish a good order in the online car-hailing industry.

Based on the analysis of equilibrium results, this paper puts forward supervision suggestions for the government from two aspects of positive and negative incentives. In terms of positive incentives, the government can use incentives, publicity and other means to encourage the construction of online car-hailing platforms to improve supervision. In terms of negative incentives, the government can use measures such as punishment and legislation to clarify responsibilities to deter online car-hailing platforms and promote their supervision.

This paper constructs a static game model of complete information, so it does not consider the situation of incomplete information which is closer to reality. In the future, we can consider the three-party game under incomplete information, and design a more effective reward and punishment mechanism for the government and online car-hailing platform, so as to further improve the supervision status of online car-hailing industry and promote social harmony and steady development.

\section{Conflicts of Interest}

The author declares no conflicts of interest regarding the publication of this paper.

\section{References}

[1] Zhao, Y.Y. (2016) Suggestions on Legislation to Regulate the System of “Car-Hailing”. China Business, 179-180.

[2] iiMedia Research. (2016) China Car-Hailing New Deal Market Impact Monitoring Report. 2-5.

[3] Li, J. and Wang, Z. (2017) Online Car-Hailing Dispatch: Deep Supply-Demand Gap Forecast on Spark. IEEE International Conference on Big Data Analysis, Beijing, 10-12 March 2017. https://doi.org/10.1109/ICBDA.2017.8078750

[4] The Ministry of Education. (2016) Report of Chinese Language Living Condition. 236.

[5] Mao, S.L. (2016) Whether the Legalization of Online Car-Hailing Can Eradicate the "Black Car" Chaos. People's Tribune, 60-62.

[6] Gao, Y., An, J. and Quan, Y.X. (2016) The Impact of Online Car Rental on Travel Mode Selection and Traffic Operation. Urban Transport of China, 1-8. 
[7] Wang, J. (2016) Safety Risk Analysis and Avoidance of Car-Hailing. Journal of Hubei University of Science, 10-14.

[8] Wang, J. (2016) Regulatory Difficulties and Solutions of China's Car-Hailing System. Administrative Law Review, 49-59.

[9] Xue, Z.Y. (2016) Study on the Quantity Control of Car-Hailing. Theory and Reform, 108-113.

[10] Mei, J. (2016) Research on the Regulatory Paradigm of China's Car-Hailing System. China Market, 163-165.

[11] Zhang, Y.N. (2016) The Supervision Perfection and Prospect of the Online Car-Hailing Industry. Enterprise Reform and Management, 59-65.

[12] Chen, S.M. (2007) The Nash Equilibrium Solution of Tripartite Game. The Science Education Article Collects, 136.

[13] Zhang, W.Y. (2012) Game Theory and Information Economics. Shanghai People's Publishing House, 61-63. 